\title{
The changing role of ultrasonography examination in patients with rheumatoid arthritis in light of recent studies
}

\author{
Marta Dura ${ }^{1,2}$, Paweł Żuchowski ${ }^{3}$, Przemysław Gorgolewski ${ }^{1}$, Marzena Waszczak-Jeka ${ }^{4}$, \\ Stawomir Jeka ${ }^{2,3}$ \\ ${ }^{1}$ Department of Radiology and Imaging Diagnostics, Dr Jan Biziel University Hospital No. 2 in Bydgoszcz, Poland \\ 2Ludwik Rydygier Collegium Medicum in Bydgoszcz, Nicolaus Copernicus University in Toruń, Poland \\ ${ }^{3}$ Department of Rheumatology and Connective Tissue Diseases, Dr Jan Biziel University Hospital No. 2 in Bydgoszcz, Poland \\ ${ }^{4}$ Non-public Health Care Centre "Nasz Lekarz", Toruń, Poland
}

\begin{abstract}
For many years, ultrasonography (US) has been a widely accepted modality used for joint assessment in patients with rheumatoid arthritis (RA). Given the efficacy of present day therapies, there is scepticism whether it is required in the first place. The most recent clinical Targeting synovitis in Early Rheumatoid Arthritis (TaSER) and Clinical Tight Control Therapy (ARCTIC) trials' results appear to contradict it, yet this does not necessarily mean ultrasound has no place in modern rheumatology. The possibility of detecting inflammation at a subclinical level carries a tremendous predictive value, enabling identification of patients likely to experience an exacerbation in the coming months. Therefore, US should be a part of the decision-making process regarding treatment modification or introduction of any additional interventions, such as glucocorticosteroid injections. The results of the most recent clinical trials do not negate the usefulness of US, but merely suggest that it ought to be used in moderation.
\end{abstract}

Key words: rheumatoid arthritis, inflammation, ultrasonography, synovitis.

\section{Introduction}

Rheumatoid arthritis (RA) is a chronic disease that leads to joint damage through inflammation of the synovial membrane [1, 2], though symptoms outside the joints may also occur in the course of RA. In the first weeks of the disease, the inflammatory process usually involves small joints of the hands and feet [3].

Nowadays early diagnosis is of greater importance than ever before. Furthermore, modern therapies and the widely used treat-to-target (T2T) strategy make it possible to achieve low disease activity and even stable remission within a short period. Early disease detection, optimally within three months, i.e. during the so-called window of opportunity, is associated with a better prognosis and more likely remission [4]. Prompt achievement of remission is associated with halted radiographic progression, in turn preventing rapid progression of disability.
Regrettably, early detection of RA still presents a major challenge in routine medical practice as the shorter the duration of the disease, the less specific its manifestation is. The sensitivity of the current 2010 American College of Rheumatology (ACR)/European League Against Rheumatism (EULAR) criteria dramatically decreases for RA, lasting less than three months. During this period, it reaches $74 \%$ at most [5]. This means that on average every fourth patient remains undiagnosed during the window of opportunity.

This may not have been a major problem in the 1990s, but today, with all the effective biological and innovative therapies at our disposal, it is of utmost importance to diagnose RA at an early stage [4]. Therefore, it is imperative to change the diagnostic algorithm in the current classification criteria.

Over the last two decades, in addition to the evolution of therapies, there have been major advances 
in the knowledge of RA. One could hazard to say that the essence of the condition is synovitis [6]. As a natural consequence of such understanding of the disease, the importance of diagnostic imaging which allows for a thorough assessment of the synovial membrane and joints, inter alia, increases. For years, the use of magnetic resonance imaging (MRI) and ultrasonography (US) has been a standard of care not only in clinical trials but also, increasingly more often, in real-world clinical practice.

Ultrasonographic imaging includes both grayscale US (GSUS), which enables assessment of conditions such as synovial hypertrophy, joint effusion or joint erosions, and power Doppler US (PDUS), which enables assessment of active synovial inflammation (synovitis).

In the last two decades, the availability of high-end US machines in conjunction with the revolution in RA treatment has brought about an unprecedented increase in publications on RA diagnosis and RA treatment monitoring. Many investigators hope that US will be included in future classification criteria.

Unfortunately, in retrospect, one can see that this is a highly difficult task, as evidenced by the work done by the EULAR-OMERACT Ultrasound Task Force established in 2004 [6, 7]. Despite the progress that has been made in recent years, there remains a need for further research in this area $[8,9]$.

The aim of the study was to evaluate the role of US testing in RA management with regards to conclusions of the Aiming for Remission in Rheumatic Arthritis: a randomized trial evaluating the benefit of ultrasound in Clinical Tight Control Therapy (ARCTIC) and Targeting synovitis in Early Rheumatoid Arthritis (TaSER) [10, 11] trials. Both trials failed to demonstrate the benefits of using a US test to monitor patients treated according to the T2T strategy, with the result that the usefulness of a US test in patients with RA may be compromised [10-12].

\section{Material and methods}

A systematic literature review was conducted using the PubMed and Cochrane Library databases to find studies on employing musculoskeletal US (MSUS) in diagnostics and monitoring of RA. The search strategy was based on the following key words: rheumatoid arthritis and ultrasound and their relevant synonyms (early rheumatoid arthritis and musculoskeletal US). Only works published after 2015 were considered. Studies with results relating to the ARCTIC and TASER studies were selected for analysis.

\section{Discussion}

Imaging diagnostics provides clinicians with a host of additional information. However, in some cases cli- nicians end up overusing it. The marked increase in the number of computed tomography (CT) tests [13] is the best example of this.

The US tests are not as closely evaluated for their usefulness as CT studies as they do not involve exposure of the patient to ionizing radiation. Therefore, we should consider whether we should make extensive use of US tests in the management of RA. Ultrasonographic testing in rheumatology has been in place for many years, since long before the onset of the biological treatment era. With the efficacy of modern biological drugs being much higher than previous therapies [14], perhaps the US test ought to be performed only in specific cases instead of on a routine basis.

The key to demonstrating the usefulness of a US test may lie in identifying patients and clinical situations in which it could have a tangible impact on further treatment. There are a number of reasons why a US test should be used selectively.

In routine clinical practice, the time a doctor can devote to each patient is limited. It stands to reason it can be hardly expected for a large number of joints to be evaluated through US testing due to its time-consuming nature. Therefore, many scientific studies have attempted to identify joints that could be considered the most representative in the course of RA.

Hand joints, in particular the metacarpophalangeal (MCP) and wrist joints, were most likely the most common choice, with the mean count of joints assessed ranging from several to a dozen or so [15-20]. Hand joints are an obvious choice as MCP joints are the most frequently affected by inflammation [19].

The standard GSUS and PDUS assessments are performed using a semi-quantitative scale. Thus, it can be considered subjective. This is another concern that, in our opinion, is related to the attempt to use US research on a broad scale. This can doubtless be eliminated through appropriate training of physicians performing ultrasound examinations. Regrettably, even with extensive experience of the performing physician, interpretation of the US examination may pose certain problems, particularly in the course of patient monitoring when radiographic progression may result in confluent erosions [21].

Ultrasonography is not the only imaging technique used to diagnose and monitor RA. It can be compared to MRI, which allows for far more accurate imaging and overall assessment, including assessment for osteomyelitis [22, 23]. However, MRI takes a longer time to complete and is more expensive due to the use of contrast media for the best possible sensitivity of imaging [22]. Therefore, it cannot be considered the examination of choice or one to be employed during routine visits. 
In this respect, US is superior to MRI and one should bear in mind that it may become the examination of choice in the diagnostic algorithm that provides for imaging examinations [24]. It is also important to note that the advantages of US testing include the ability to evaluate more joints than MRI and evaluate structures in a dynamic study [25]. Additionally, new techniques, such as elastography, increase diagnostic sensitivity in locomotor system diseases, e.g. in carpal tunnel syndrome [26].

Whenever a new diagnostic modality is introduced, it should always be compared to the currently used method. For many years, the disease activity score (DAS/ DAS28) has been used for the monitoring of RA activity. It includes physical examination for tender and swollen joints, laboratory parameters and general assessment of the patient's health status. Unfortunately, although designed for early RA (ERA) diagnosis, the DAS is also used in patients with long-standing RA (LSRA) [27].

This leads to situations in which pain resulting from destructive lesions rather than active inflammation or joint swelling stemming from irreversible synovial hypertrophy due to long-term inflammation is taken into account [27]. On the other hand, physical examination cannot detect subclinical inflammatory lesions [27]. Therefore, the correlation between US findings and DAS28 scores tends to be weak. However, one should bear in mind that this results from differences in assessment methods rather than the superiority of one method over the other.

Moreover, it is worth noting that a certain flow within the synovial membrane may also be observed in individuals with no inflammatory diseases. Therefore, it is necessary to determine the limit from which the PD signal will actually be associated with inflammation within the synovial membrane [28]. This is an extremely important issue, especially when using such sensitive techniques as superb microvascular imaging (SMI) in the assessment of the synovial membrane [29].

A better correlation between US and clinical assessment is achieved using measures such as the simplified disease activity index (SDAI) and clinical disease activity index (CDAI) [30-32].

It is worth noting a single yet salient advantage of the US test over the clinical evaluation methods mentioned above. A US examination of aching joints can help determine the cause of pain that can originate from irreversible destructive changes or active inflammation of the synovial membrane [33]. Such information, from a clinician's point of view, may significantly impact further therapeutic decisions.

\section{Unmet expectations}

Given the time and resources required to introduce US into routine diagnostics in rheumatology, opposition to it should not come as a surprise [12]. Nevertheless, taking a critical view, one should consider whether "unmet expectations" are a consequence of too high a hope regarding the use, and overuse, of US. Yet even detractors point to the fact that US is far more accurate than physical examination, having much higher sensitivity in detecting RA-specific lesions [12].

It is worth remembering at this point that RA is a disease affecting a relatively small portion of the population - about $1.5 \%$ of the general population. Consequently, to gather a large group of RA patients and provide them with a long-term follow-up is a task that is far from easy, and if we take into account differences in disease duration, possible complications and therapies, a reliable study becomes a challenge. Therefore, a great many publications concern observation studies involving only up to several dozen subjects.

Thus, especial significance is placed on the results of two studies: TaSER and ARCTIC [10, 12, 34]. The studies included 111 and 238 subjects, respectively. The common feature of both trials was their research hypothesis. The aim was to determine whether including MSUS in the assessment of clinical efficacy of T2T therapy significantly improves its efficacy compared with T2T based on standard assessment of the patient's health status $[34,35]$. Both studies failed to demonstrate increased efficacy of T2T therapy with MSUS [10, 34]. Nevertheless, it would be difficult to agree with the opinion that the results of both trials are disappointing in terms of MSUS use [12].

The efficacy of aggressive T2T therapies is so high that the application of extended diagnostic assessment may indeed prove to be of marginal importance. If true, one could conclude that imaging examinations are overrated and overused, which would not be a first in modern medicine, especially in conventional radiology [13]. Diagnostic imaging should not be a tool used in obvious situations, nor should it replace physical examination. Perhaps this is the reason for the disappointment - the use of MSUS was considered where it is simply redundant. The main conclusion from ARCTIC and TASER one should arrive at is that the T2T strategy is highly effective, which is also confirmed by ultrasound.

The ARCTIC and TaSER studies enrolled patients with RA in whom aggressive T2T treatment had been initiated $[10,11]$. The conclusions of both studies ought to draw one's attention to the following facts:

- in ERA patients, pain is usually associated with an active inflammatory process in the synovial membrane and not with destructive changes in joints,

- the efficacy of modern biological drugs and the T2T treatment strategy has been confirmed by multiple clinical studies. 
Thus, it raises the question whether these studies are a case of US examination overuse. Considering the carefully selected group of patients and the conclusions of both studies, one might fail to see a reasoning for conducting US examination. Naturally, one may come to such an opinion not until after perusing the ARCTIC and TaSER trials, which in our opinion makes the studies highly valuable.

Clinical trials feature very strict patient inclusion criteria, and the protocol is closely observed throughout the entire study. However, routine clinical practice tends to be strikingly different. The conclusions from the Corrona registry are worth bringing up for consideration [35]. The analysis included 5,546 patients, of whom 1,018 (18.7\%) underwent MSUS during the visits. Higher rates of patients with low disease activity and remission were reported for the MSUS group (64.9\% vs. $56.8 \%, p<0.01$ ) [35]. The Corrona registry data are collected in routine clinical practice settings and the patient population is much more diverse than in the aforementioned clinical trials.

\section{Results - selective application of ultrasonography testing}

The lack of requirements for ultrasound scans in the course of aggressive T2T therapy does mean that they are impractical in the diagnosis and monitoring of RA patients. This has been proven by the ARCTIC study [10], for instance. In its course, a large amount of data was collected which allowed for a comprehensive analysis of various aspects of MSUS use in RA patients.

Thanks to novel therapies, radiographic progression is very slow. Nevertheless, it plays a key role in compounding patients' disability and impairing their social functions. As such, this component of the disease should not be neglected, even if patients achieve remission and their

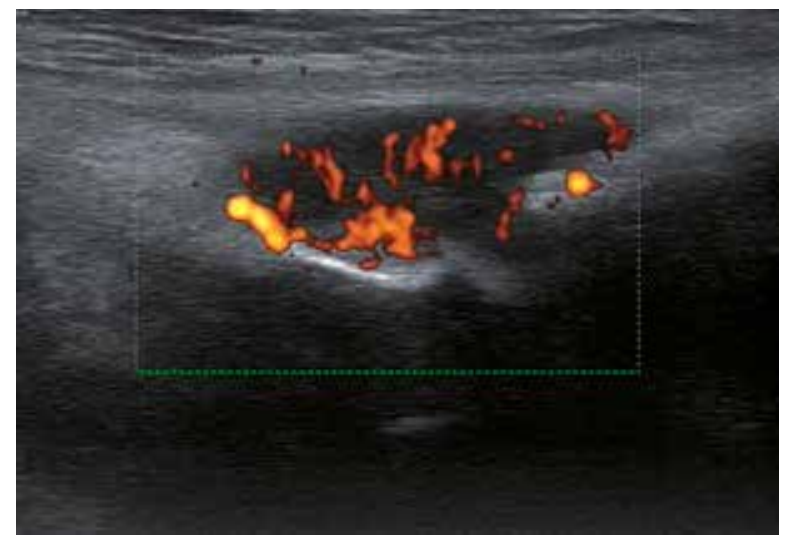

Fig. 1. MTP III joint with PD synovitis (grade 3) in patient with rheumatoid arthritis. general health status is good. An analysis of the ARCTIC data showed the highest odds ratio for no yearly radiographic progression in patients with remission observed on ultrasound [36]. By all means, this is not equivalent to a good combined outcome, which also includes physical function. However, it should be emphasized once again that MSUS should not be considered an examination enabling an overall assessment of patients' health status. There are a number of other parameters that affect the health status of patients with RA - the authors themselves draw attention to this fact [36].

In rheumatoid arthritis, glucocorticosteroid (GCS) injections are also regularly administered. An analysis of this type of intervention in the ARCTIC study showed that better outcomes were achieved when the decision on injections was based on PDUS rather than physical examination [37]. The joint swelling found during physical examination did not play a major role in the procedure's effectiveness. The same analysis also showed that the use of US for guiding the injection had no significant effect on its efficacy [37]. With experienced clinicians, the palpation-guided procedure is sufficient [37].

If remission is achieved, dose reduction is usually considered, especially in the case of biologic therapies and subsequently disease-modifying antirheumatic drugs (DMARDs), which are used as combination therapies in active, often aggressive diseases, and constitute a high burden. The use of PDUS in this setting may identify patients with subclinical inflammation. This is associated with a higher risk of disease exacerbation following dose reduction or treatment discontinuation in the following months $[38,39]$. The use of PDUS in such circumstances may also reduce RA treatment costs by indicating a group of patients in whom treatment modification is not associated with an increased risk of exacerbation [39]. In Figures 1 and 2 active synovitis and remission in PDUS are presented.

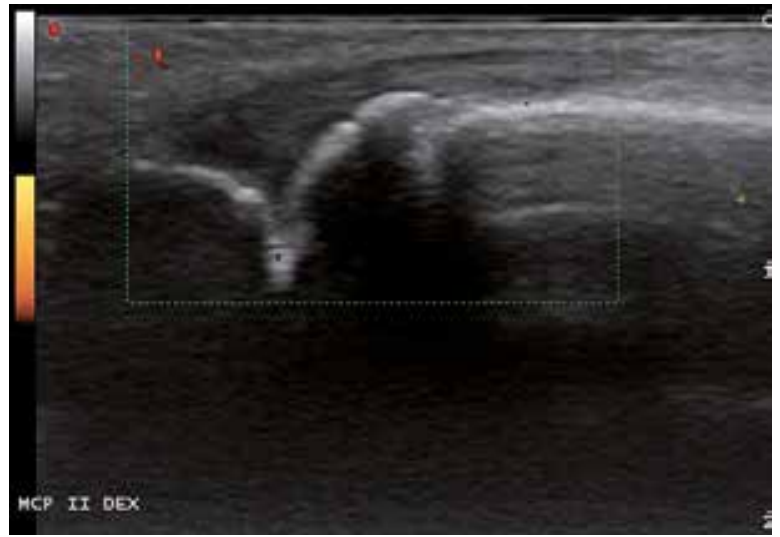

Fig. 2. MCP II joint without synovitis in patient with rheumatoid arthritis. 
On the other hand, the lack of a PD signal is strongly correlated with the lack of radiological change progression in RA patients $[36,40]$. Such information should not be underestimated from a clinical point of view. Considering that the goal of the treatment is to arrest disease progression, it might be advisable to add the lack of signal in PDUS examination to remission criteria [40].

Ultrasound offers a clear advantage in assessing subclinical synovitis, which is important for the evaluation of radiographic progression risk and for the identification of patients who can maintain remission despite dose reduction [1, 41, 42]. Moreover, in ERA diagnosis, US allows for early detection of the disease and should be included in future classification criteria [43-45]. The application of a US is shown in Table I.

The results of the latest clinical trials with aggressive T2T therapies show that the efficacy of current therapies is very high [14]. Perhaps in the case of such an effective treatment, in which no significant therapeutic decisions are made, a US examination is unnecessary. The evidence for this can be the conclusions drawn from two independent studies extensively quoted in this paper, i.e. TASER and ARCTIC $[10,46]$.

However, US testing may provide added value in cases that raise diagnostic doubts among experienced clinicians, e.g. when deciding whether to reduce drug dosage, and on possible interventions, e.g. injections of GCS [37].

\section{Conclusions}

The musculoskeletal US examination ought to be considered instrumental in neither diagnostics nor monitoring of rheumatoid arthritis. Like other types of imaging examination, it has its limitations. In rheumatoid arthritis, only the radiographic course of the disease is provided, which is not the sole parameter affecting the assessment of a patient's health status. In the light of the published research results, we should review our opinion on the role of US in rheumatoid arthritis. Perhaps US should not be used on a regular basis but only in situations where clinical assessment is not sufficient to make further treatment decisions.

It is our opinion that the current analysis suggests that a certain framework of US examination use has been given - on one hand, the usefulness of US testing in the aggressive T2T strategy has yet to be proven; on the other, when deciding on interventions, or alterations and, in particular, reduction of drug dosage, US examination allows clinicians to be provided with key information.

We believe that US examination has not only retained its value in rheumatoid arthritis management
Table I. Application of an ultrasonography test

\begin{tabular}{|l|}
\hline Confirmed usefulness \\
\hline Early diagnosis of RA \\
\hline $\begin{array}{l}\text { A decision on a possible change of therapy: } \\
\text { assessment of RA activity at subclinical level }\end{array}$ \\
\hline $\begin{array}{l}\text { Diagnosis of the causes of joint pain (destructive changes/ } \\
\text { active inflammatory process) }\end{array}$ \\
\hline Injection decisions \\
\hline Doubtful usefulness \\
\hline Monitoring of aggressive T2T treatment strategy \\
RA-rheumatoid arthritis, T2T-treat-to-target strategy.
\end{tabular}

but is an exceptionally sensitive instrument, and, if used selectively, can facilitate proper judgment in routine clinical practice. Its role has simply changed as treatment efficiency has increased. There is increasing evidence that US examination is the most beneficial whenever diagnostic uncertainties arise, i.e. when a firm diagnosis proves difficult, or there is a need to alter the existing treatment.

The authors declare no conflict of interest.

\section{References}

1. do Prado AD, Staub HL, Bisi MC, et al. Ultrasound and its clinical use in rheumatoid arthritis: where do we stand? Adv Rheumatol 2018; 58: 19.

2. Grassi W, De Angelis R, Lamanna G, Cervini C. The clinical features of rheumatoid arthritis. Eur J Radiol 1998; 27 (Suppl 1): 18-24, DOI: 10.1016/s0720-048x(98)00038-2.

3. Heidari B. Rheumatoid arthritis: early diagnosis and treatment outcomes. Caspian J Intern Med 2011; 2: 161-170.

4. Burmester GR, Pope JE. Novel treatment strategies in rheumatoid arthritis. Lancet 2017; 389: 2338-2348, DOI: 10.1016/ S0140-6736(17)31491-5.

5. Migliore A, Bizzi E, Petrella L, et al. The challenge of treating early-stage rheumatoid arthritis: the contribution of mixed treatment comparison to choosing appropriate biologic agents. BioDrugs 2016; 30: 105-115, DOI: 10.1007/s40259016-0164-7.

6. Jeka S, Dura M, Zuchowski P, et al. The role of ultrasonography in the diagnostic criteria for rheumatoid arthritis and monitoring its therapeutic efficacy. Adv Clin Exp Med 2018; 27: 13031307, DOI: 10.17219/acem/69133.

7. Naredo E, Wakefield RJ, lagnocco A, et al. The OMERACT ultrasound task force-status and perspectives. J Rheumatol 2011; 38: 2063-2067, DOI: 10.3899/jrheum.110425.

8. D’Agostino MA, Terslev L, Aegerter P, et al. Scoring ultrasound synovitis in rheumatoid arthritis: a EULAR-OMERACT ultrasound taskforce - part 1: definition and development of a standardised, consensus-based scoring system. RMD Open 2017; 3: e000428, DOI: 10.1136/rmdopen-2016-000428. 
9. Terslev L, Christensen R, Aga AB, et al. Assessing synovitis in the hands in patients with rheumatoid arthritis by ultrasound an agreement study exploring the most inflammatory active side from two Norwegian trials. Arthritis Res Ther 2019; 21: 166, DOI: 10.1186/s13075-019-1930-y.

10. Haavardsholm EA, Aga AB, Olsen IC, et al. Ultrasound in management of rheumatoid arthritis: ARCTIC randomised controlled strategy trial. BMJ 2016; 354: i4205, DOI: 10.1136/bmj. i4205.

11. Dale J, Stirling A, Zhang R, et al. Targeting ultrasound remission in early rheumatoid arthritis: the results of the TaSER study, a randomised clinical trial. Ann Rheum Dis 2016; 75: 1043 1050, DOI: 10.1136/annrheumdis-2015-208941.

12. Caporali R, Smolen JS. Back to the future: forget ultrasound and focus on clinical assessment in rheumatoid arthritis management. Ann Rheum Dis 2018; 77: 18-20, DOI: 10.1136/annrheumdis-2017-211458.

13. Schwartz DT. Counter-Point: are we really ordering too many CT scans? West J Emerg Med 2008; 9: 120-122.

14. Gadeholt O. Forward to the past: ultrasound might be necessary in some patients with rheumatoid arthritis. Ann Rheum Dis 2019; 78: e56, DOI: 10.1136/annrheumdis-2018-213278.

15. Cerqueira M, Teixeira F, Sousa Neves J, et al. Relationship between clinical evaluation and ultrasound assessment of rheumatoid arthritis patients using a 12 joint score. Int J Rheum Dis 2013; 20: 852-858, DOI: 10.1111/1756-185X.13005.

16. Chakr RM, Mendonça JA, Brenol CV, et al. Assessing rheumatoid arthritis disease activity with ultrasound. Clin Rheumato 2013; 32: 1249-1254, DOI: 10.1007/s10067-013-2291-6.

17. Terslev L, Naredo E, Aegerter P, et al. Scoring ultrasound synovitis in rheumatoid arthritis: a EULAR-OMERACT ultrasound taskforce - part 2: reliability and application to multiple joints of a standardised consensus-based scoring system. RMD Open 2017; 3: e000427, DOI: 10.1136/rmdopen-2016-000427.

18. D'Agostino MA, Terslev L, Wakefield R, et al. Novel algorithms for the pragmatic use of ultrasound in the management of patients with rheumatoid arthritis: from diagnosis to remission. Ann Rheum Dis 2016; 75: 1902-1908, DOI: 10.1136/annrheumdis-2016-209646.

19. Grassi W, Gaywood I, Pande I, et al. From DAS 28 to SAS 1. Clin Exp Rheumatol 2012; 30: 649-651.

20. Yoshimi R, Ihata A, Kunishita Y, et al. A novel 8-joint ultrasound score is useful in daily practice for rheumatoid arthritis. Mod Rheumatol 2014; 25: 379-385, DOl: 10.3109/14397595.2014.974305.

21. Szkudlarek M, Terslev L, Wakefield RJ, et al. Summary findings of a systematic literature review of the ultrasound assessment of bone erosions in rheumatoid arthritis. J Rheumatol 2016; 43: 12-21, DOI: 10.3899/jrheum.141416.

22. Sudoł-Szopińska I, Jans L, Teh J. Rheumatoid arthritis: what do MRI and ultrasound show. J Ultrason 2017; 17: 5-16, DOI: 10.15557/JoU.2017.0001.

23. Ohrndorf S, Boer AC, Boeters DM, et al. Do musculoskeletal ultrasound and magnetic resonance imaging identify synovitis and tenosynovitis at the same joints and tendons? A comparative study in early inflammatory arthritis and clinically suspect arthralgia. Arthritis Res Ther 2019; 21: 59, DOI: 10.1186/ s13075-019-1824-z.
24. Huajun X, Yingchun Z, Huimei Z, et al. Comparison of the clinical effectiveness of US grading scoring system vs MRI in the diagnosis of early rheumatoid arthritis (RA). J Orthop Surg Res 2017; 12: 152, DOI: 10.1186/s13018-017-0653-5.

25. Tan YK, Østergaard M, Conaghan PG. Imaging tools in rheumatoid arthritis: ultrasound vs magnetic resonance imaging. Rheumatology (Oxford) 2012; 51 (Suppl 7): vii36-42, DOI: 10.1093/rheumatology/kes329.

26. Cingoz M, Kandemirli SG, Alis DC, et al. Evaluation of median nerve by shear wave elastography and diffusion tensor imaging in carpal tunnel syndrome. Eur J Radiol 2018; 101: 59-64, DOI: 10.1016/j.ejrad.2018.02.005.

27. Barczyńska TA, Dura M, Blumfield E, et al. DAS28 score vs. ultrasound examination for assessment of rheumatoid arthritis disease activity: comparison and discussion of pros and cons. Reumatologia 2015; 53: 213-218, DOI: 10.5114/ reum.2015.53999.

28. Padovano I, Costantino F, Breban M, et al. Prevalence of ultrasound synovial inflammatory findings in healthy subjects. Ann Rheum Dis 2016; 75: 1819-1823, DOI: 10.1136/annrheumdis-2015-208103.

29. Yu X, Li Z, Ren M, et al. Superb microvascular imaging (SMI) for evaluating hand joint lesions in patients with rheumatoid arthritis in clinical remission. Rheumatol Int 2018; 38: 18851890, DOI: 10.1007/s00296-018-4112-3.

30. D'Agostino MA, Boers M, Wakefield RJ, et al. Exploring a new ultrasound score as a clinical predictive tool in patients with rheumatoid arthritis starting abatacept: results from the APPRAISE study. RMD Open 2016; 5: e000237, DOI: 10.1136/ rmdopen-2015-000237.

31. Gärtner M, Mandl P, Radner H, et al. Sonographic joint assessment in rheumatoid arthritis: associations with clinical joint assessment during a state of remission. Arthritis Rheum 2013; 65: 2005-2014, DOI: 10.1002/art.38016.

32. Olmez MO, Gunal EK, Ureyen SB, et al. Comparison of composite indices with global synovitis score on ultrasound for detecting remission. Clin Rheumatol 2017; 37: 1111-4, DOI: 10.1007/s10067-017-3925-x.

33. Jeka S, Dura M, Żuchowski P, et al. The role of ultrasonography in monitoring long-standing rheumatoid arthritis: a pilot study. Reumatologia 2017; 55: 177-182, DOI: 10.5114/ reum.2017.69781.

34. Dale J, Stirling A, Zhang R, et al. Targeting ultrasound remission in early rheumatoid arthritis: the results of the TaSER study, a randomised clinical trial. Ann Rheum Dis 2016; 75: 10431050, DOI: 10.1136/annrheumdis-2015-208941.

35. Pappas DA, Saunders KC, Etzel CJ, et al. Evaluation of the use of ultrasound to manage patients with rheumatoid arthritis over time: results from the Corrona registry. Ann Rheum Dis 2018; 77: 906-907.

36. Paulshus Sundlisæter N, Aga AB, Olsen IC, et al. Clinical and ultrasound remission after 6 months of treat-to-target therapy in early rheumatoid arthritis: associations to future good radiographic and physical outcomes. Ann Rheum Dis 2018; 77: 1421-1425, DOI: 10.1136/annrheumdis-2017-212830.

37. Nordberg LB, Lillegraven S, Aga AB, et al. The impact of ultrasound on the use and efficacy of intraarticular glucocorticoid injections in early rheumatoid arthritis: secondary analyses 
from a randomized trial examining the benefit of ultrasound in a clinical tight control regimen. Arthritis Rheumatol 2018; 70: 1192-1199, DOI: 10.1002/art.40494.

38. Naredo E, Valor L, De la Torre I, et al. Predictive value of doppler ultrasound-detected synovitis in relation to failed tapering of biologic therapy in patients with rheumatoid arthritis. Rheumatology (Oxford) 2015; 54: 1408-1414, DOI: 10.1093/rheumatology/kev006.

39. Simpson E, Hock E, Stevenson $M$, et al. What is the added value of ultrasound joint examination for monitoring synovitis in rheumatoid arthritis and can it be used to guide treatment decisions? A systematic review and cost-effectiveness analysis. Health Technol Assess 2018; 22: 1-258, DOI: 10.3310/hta22200.

40. de Miguel E, Pecondón-Español A, Castaño-Sánchez M, et al. A reduced 12 -joint ultrasound examination predicts lack of $\mathrm{X}$-ray progression better than clinical remission criteria in patients with rheumatoid arthritis. Rheumatol Int. 2017; 37: 1347-1356, DOI: 10.1007/s00296-017-3714-5.

41. Han J, Geng Y, Deng X, et al. Subclinical synovitis assessed by ultrasound predicts flare and progressive bone erosion in rheumatoid arthritis patients with clinical remission: a systematic review and metaanalysis. J Rheumatol 2016; 43: 2010-2018, DOI: 10.3899/jrheum.160193.
42. Naredo E, Valor L, De la Torre I, et al. Predictive value of Doppler ultrasound-detected synovitis in relation to failed tapering of biologic therapy in patients with rheumatoid arthritis. Rheumatology (Oxford) 2015; 54: 1408-1414, DOI: 10.1093/ rheumatology/kev006.

43. Ponikowska M, Świerkot J, Nowak B. The importance of ultrasound examination in early arthritis. Reumatologia 2018; 56 : 354-361, DOI: 10.5114/reum.2018.80712.

44. Freeston JE, Wakefield RJ, Conaghan PG, et al. A diagnostic algorithm for persistence of very early inflammatory arthritis: the utility of power Doppler ultrasound when added to conventional assessment tools. Ann Rheum Dis 2010; 69: 417-419, DOI: 10.1136/ard.2008.106658.

45. Ten Cate DF, Luime JJ, Swen N, et al. Role of ultrasonography in diagnosing early rheumatoid arthritis and remission of rheumatoid arthritis - a systematic review of the literature. Arthritis Res Ther 2013; 15: R4, DOI: 10.1186/ar4132.

46. Dale J, Stirling A, Zhang R, et al. Targeting ultrasound remission in early rheumatoid arthritis: the results of the TaSER study, a randomised clinical trial. Ann Rheum Dis 2016; 75: $1043-$ 1050, DOI: 10.1136/annrheumdis-2015-208941. 